\title{
Tratamiento odontológico en la displasia ectodérmica. Actualización
}

\author{
Pipa Vallejo A*, López-Arranz Monje E*, González García M**
}

\section{RESUMEN}

La displasia ectodérmica hidrótica es un síndrome congénito caracterizado por hipotricosis, hiperqueratosis palmoplantar y distrofia ungueal, que no va acompañada de transtornos de la sudoración. Se expone el caso de una niña con displasia ectodérmica hidrótica con oligodoncia y reabsorción de procesos alveolares a la que se le realiza una rehabilitación protésica con prótesis removible de acrílico, consiguiendo una estética, funcionalidad y adaptación excelentes así como una considerable mejora de la autoestima.

Palabras clave: Displasia ectodérmica hidrótica, oligodoncia, rehabilitación.

\section{SUMMARY}

Hidrotic ectodermal dysplasia is a congenital syndrome characterized by hypotrichosis, palmoplantar hyperkeratosis and fingernail dystrophy, not presenting sweating disorders. Here we review the case of a girl patient with hidrotic ectodermal dysplasia with oligodontia and reabsortion of the aveolar processus, to whom a prothesic rehabilitation was performed with the collocation of the removable acrylic prostheses, obteining an excellent aesthetic resoult and excellent adaptation as will as considerable improvement in her self-steem.

Key words: Hidrotic ectodermal dysplasia, oligodontia, rehabilitation.

Aceptado para publicación: Mayo 2005.

* $\quad$ Escuela de Odontología. Facultad de Medicina. Universidad de Oviedo.

** Servicio de Cirugía Oral y Maxilofacial del Hospital Universitario Central de Asturias.

Pipa Vallejo A, López-Arranz Monje E, González García M. Tratamiento odontológico en la displasia ectodérmica. Actualización. Av. Odontoestomatol 2006; 22 (3): 171-176.

\section{INTRODUCCIÓN}

La displasia ectodérmica forma parte de un amplio grupo de enfermedades o síndromes que tienen en común evidenciar defectos en el normal desarrollo de dos o más tejidos derivados del ectodermo. Son genodermatosis congénitas difusas, no progresivas, caracterizadas por la carencia o escasa presencia de pelo, dientes, uñas y glándulas sudoríparas ecrinas, a las que se pueden añadir anomalías en la configuración externa (nariz, pabellones auriculares, labios), trastornos del SNC, ojos, mucosa naso bucal y melanocitos. ${ }^{1}$

La displasia ectodérmica es un desorden relativamente raro con una frecuencia que varía entre 
1:10.000 y 1:100.000 niños nacidos vivos y es más frecuente en varones. En la mayoría de los casos es de herencia autosómica recesiva, pero puede ser también autosómica dominante o ligada al cromosoma X.

Los tipos de displasia ectodérmica que se observan más frecuentemente son: los hipohidróticoanhidróticos y los hidróticos.

El tipo hipohidrótico-anhidrótico, síndrome de ChristSiemens-Touraine, fue descrito en 1848 por Thurman y se caracteriza por la tríada hipotricosis (anomalías en piel, pelo y uñas), hipodoncia o anodoncia e hipohidrosis (ausencia parcial o total de glándulas sudoríparas ecrinas) y algunos rasgos como: abombamiento de la frente, nariz en silla de montar, labios evertidos, etc.

El tipo hidrótico es definido en 1929 por Clouston y se distingue por hipotricosis, distrofia ungueal e hiperqueratosis de palmas de las manos y plantas de los pies.

En las displasias ectodérmicas se pueden dar numerosas combinaciones de las alteraciones clínicas, observándose síndromes variados y hasta 154 tipos diferentes de displasia ectodérmica y 11 subgrupos, que según se afecten pelo, dientes, uñas o glándulas sudoríparas se denominan desde el 1 al $4 .{ }^{2}$

Recientemente ha sido propuesta una nueva clasificación de DE basada en alteraciones de las funciones moleculares proteicas que están detrás. ${ }^{3}$

El diagnóstico de los pacientes con displasia ectodérmica está basado, fundamentalmente, en la historia clínica (distrofia ungueal, hipotricosis, anodoncia, oligodoncia, hipodoncia, etc.); en la realización de biopsia cutánea, en los casos de alteración de la sudación (disminución de unidades pilosebáceas y glándulas sudoríparas); estudio capilar que muestra cabellos finos y delgados; radiografía panorámica (evidenciando claramente agenesias y dismorfias dentales).

La hipodoncia, oligodoncia, o la anodoncia, es de gran interés a la hora de diagnosticar las displasias, existiendo ausencia congénita de 6 o más dientes, excluyendo los terceros molares. La oligodoncia se encuentra asociada con más frecuencia a factores hereditarios de tipo dominante, poligénico y ligado al cromosoma X.4,5 También es muy frecuente en estos pacientes el hallazgo de anomalías en la forma de los dientes, erupción retardada e inclusiones dentarias.

El diagnóstico diferencial de las displasias ectodérmicas se debe realizar con patologías como: la aplasia de cutis congénita, la disqueratosis congénita, la fiebre de origen desconocido o síndromes progeroides, como el síndrome de Werner o el de RothmundThompson. ${ }^{6}$

El pronóstico, en general, es bueno, salvo los casos con hipo o anhidrosis, que alcanzan tasas de mortalidad del $30 \%$ en la primera infancia, fundamentalmente, debido a infecciones respiratorias dada la ausencia de glándulas mucosas en el aparato respiratorio.

\section{OBSERVACIÓN CLÍNICA}

Acude a la Escuela de Odontología una niña de 36 meses, remitida por el servicio de Genética del Hospital Universitario Central de Asturias, que presenta: anodoncia parcial de incisivos y molares temporales, al igual que su madre; alopecia difusa leve en cuero cabelludo con pelo fino y sin tricorrexis, salvo en la zona cenital donde existe una alopecia más concreta derivada de tricotilomanía por tics de retorcimiento piloso.

No se observa afectación de cejas ni pestañas, ni rasgos especiales en la cara, excepto labio inferior discretamente protruido, con desarrollo psicomotor y crecimiento normal, y sin alteraciones en glándulas sudoríparas, uñas,... rasgos que tampoco aparecen alterados en su madre.

Antecedentes familiares: madre asmática y con agenesias dentales, padre con antecedentes diabéticos. Antecedentes personales: embarazo normal, parto a término eutócico instrumentado con ventosa. Lactancia materna durante 2 meses. Vacunas controladas. 
En la exploración clínica intraoral se observa atrofia ósea de los procesos alveolares tanto en el maxilar superior como en mandíbula y numerosos espacios edéntulos. Así mismo, los dientes presentes tienen formas conoides, acortadas, con aspecto de clavos.

Ortopantomografía: presenta numerosas agenesias en dentición temporal, más de la mitad de las que tendría que haber en condiciones normales (oligodoncia), retrasos en la erupción, formas dentarias anómalas, inclusiones dentarias (Fig.1).

Estudio de modelos: plano terminal de clase I, relación canina de clase I.

En conclusión, se trata de una forma leve de displasia ectodérmica hidrótica que, al menos en su expresión de agenesia de incisivos, ha sido transmitida por vía materna, tal vez autosómica dominante, sin excluir una forma ligada al cromosoma X.

Se busca dar una solución aceptable ante un caso complejo dada la poca edad de esta niña y la situación emocional materna agobiada por la numerosa ausencia de dientes en zonas tan comprometidas estéticamente.

Por otra parte, se considera iniciar el tratamiento protésico lo antes posible, por un lado para limitar la

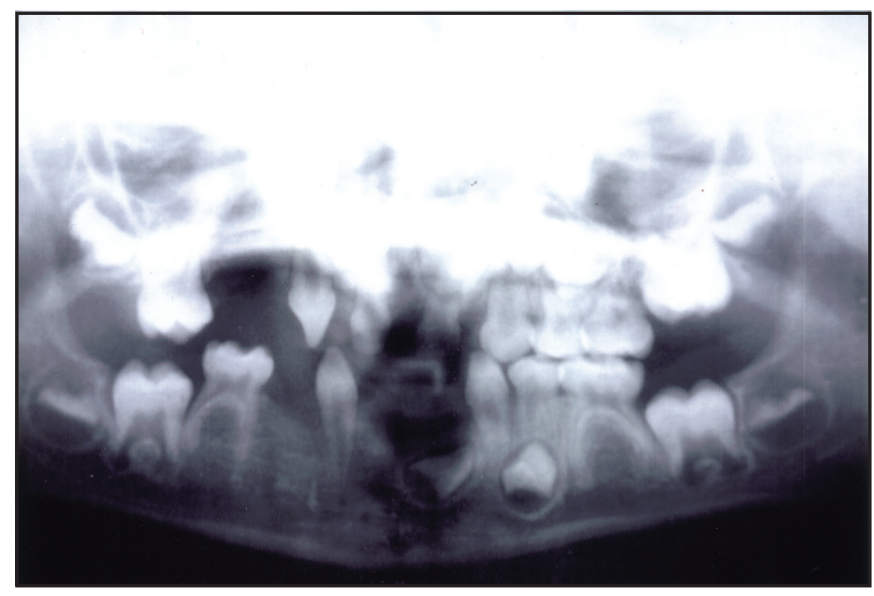

Fig. 1. Inclusión dentaria y oligodoncia. reabsorción de los procesos alveolares y por otro para minimizar el impacto psicológico y emocional que la falta de dientes puede condicionar en su vida de relación.

A la edad de 3 años existe la dificultad a la hora de tomar las impresiones, ya que no se cuenta con buena colaboración por parte de la paciente que a su edad no percibe, en este caso, la necesidad de colocar aparatología ya que, además de que puede ser molesta, le puede dificultar su vida social: juegos, deportes, etc.

Se recurre al uso de silicona pesada, que se maneja mejor, para tomar unas impresiones, hacer una cubeta individual y posteriormente poder tomar una impresión de alginato en condiciones más favorables.

Se consideró oportuno realizar una prótesis parcial de acrílico superior e inferior (Fig. 2) con unos retenedores de acero en algunos de los dientes presentes en las arcadas, que va a permitir introducir algunas modificaciones si fuera preciso y que van a dar una estética razonable, una función masticatoria aceptable al complementar las ausencias dentarias, a la vez que se intenta mantener los procesos alveolares maxilares sin atrofia y posibilitar una vida social prácticamente normal tan importante a estas edades en el desarrollo psicológico y de la personalidad del niño. ${ }^{7}$

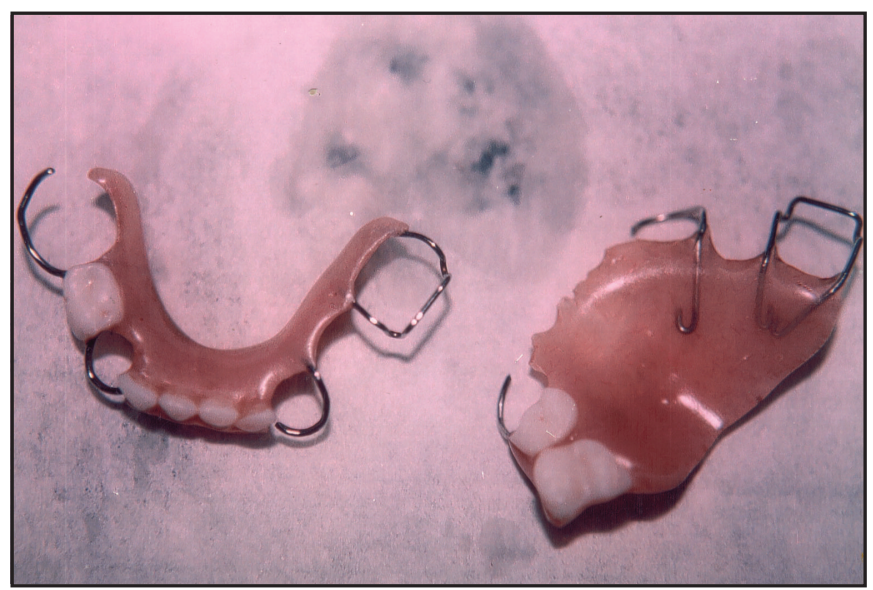

Fig. 2. 
Se insiste en la higiene que es parte fundamental en el pronóstico dental de estos pacientes, en el sentido de que le controlen el cepillado de los dientes, aporte de gotas de flúor diario según peso, control de la ingesta de productos azucarados, dulces, comidas entre horas, en la medida de lo posible y se hacen revisiones periódicas a lo largo de estos años, aproximadamente cada 6 meses, en las que se aplica gel de flúor tópico a la vez que se introducen pequeños ajustes y adaptaciones en las prótesis, sobretodo la inferior. ${ }^{8,9}$

Cinco años después, a los 8 años, al estar enferma de gastroenteritis y coincidiendo con la erupción de dientes permanentes, dismórficos, deja de ponerse las prótesis de acrílico durante dos meses.

Cuando acudió a nuestra consulta tras este periodo no fue posible ajustar las placas, por otro lado varias veces modificadas, y se opta por realizar unas nuevas prótesis removibles de acrílico (Fig. 3), dada la perfecta adaptación a las anteriores y los buenos resultados obtenidos con ellas.

En el momento actual, seguimos controlando la evolución de la erupción de las distintas piezas aún sin erupcionar, a la vez que se insiste en las medidas higiénicas de cepillado, aplicaciones tópicas de flúor cada seis meses, uso de colutorios de flúor diariamente, revisiones periódicas, etc.

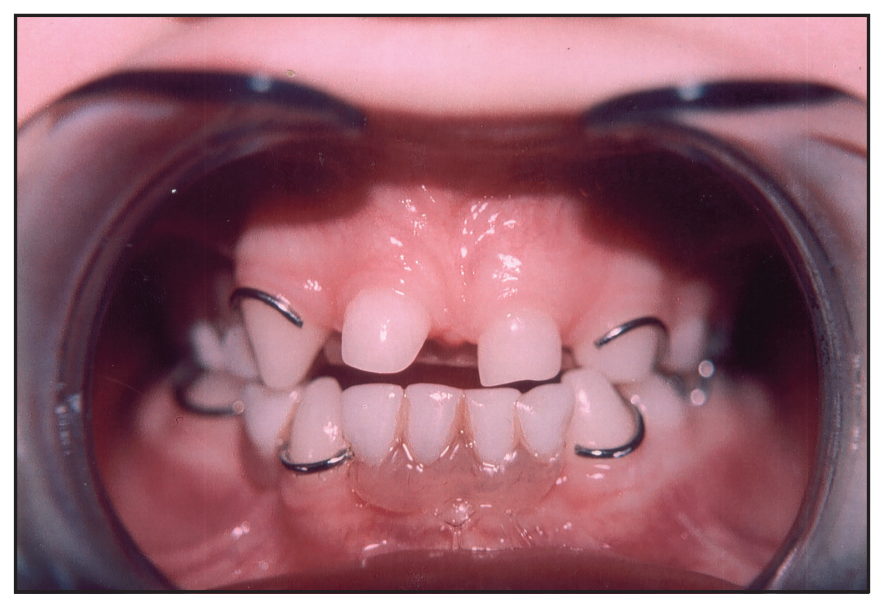

Fig. 3.

\section{DISCUSIÓN}

La displasia ectodérmica hidrótica, o síndrome de Clouston, se transmite por herencia autosómica dominante, siendo fatal si es homocigótica, puede presentar uñas distróficas, hipoplásicas o ausentes, pelo ralo, queratodermia de palmas y plantas. Dentición normal aunque, a veces, hay dientes pequeños, caries y agenesias. Sudoración normal siempre. Algunos individuos pueden presentar ausencia de cejas, pestañas e hiperqueratosis de rodillas, codos, nudillos y debajo del borde libre de las uñas.

Se pensó en un defecto molecular de la queratina, aunque posteriores estudios de Hayflick y cols., en 1996 investigando los genes de la queratina en los cromosomas 12 y 17 concluyen que no existen tales defectos en la queratina o en las proteínas asociadas a la misma.

Actualmente se relaciona la displasia ectodérmica hidrótica con una mutación en la proteína conexina Cx30 que se localiza en el locus 13q12 del gen GJB6, mutación que además va a estar en la causa de otra serie de enfermedades como el síndrome de queratitis- ictiosis- sordera, entre otros. ${ }^{10,11}$

El desarrollo físico es normal, en general, aunque se han observado casos con estatura reducida, epilepsia, sordera, dedos en palillo de tambor, poromas múltiples y cataratas bilaterales.

Desde el punto de vista odontoestomatológico se busca dar una alternativa aceptable a estos pacientes de muy corta edad y con casos complejos de ausencia congénita de dientes.

La rehabilitación prostodóncica es fundamental en estas situaciones, intentando dar una solución funcional y estética que permita realizar una vida lo más normal posible al niño sin lesionar su autoestima y su desarrollo psicológico y que la conducta no se vea afectada.

El tratamiento protésico debe hacerse de una forma individualizada y siempre a partir de un encerado de estudio que busque una buena estabilidad oclusal. 
Pipa Vallejo A, López-Arranz Monje E, González García M. Tratamiento odontológico en la displasia ectodérmica. Actualización

Debe iniciarse lo más precozmente que se pueda, para evitar en lo posible la reabsorción y atrofia de los procesos alveolares y controlar la dimensión vertical que se puede ver gravemente afectada ante la falta total o parcial de dientes.

Se han planteado por los diferentes autores distintas posibilidades de rehabilitar a estos pacientes: en general casi todos parecen estar de acuerdo en recomendar la utilización de prótesis removibles en las primeras etapas de crecimiento (3-5 años), que permitan establecer una nueva dimensión vertical o relaciones intermaxilares, para posteriormente, siempre que sea posible, optar por prótesis fijas provisionales, a la espera de que el paciente finalice el crecimiento y se pueda contar con una situación más estable y predecible y entonces plantear tratamientos con implantes. ${ }^{12}$

De ahí que contemos por lo tanto con diversas opciones rehabilitadoras, desde las soluciones convencionales y clásicas como son: prótesis completas mucosoportadas (en casos de edentulismo total, frecuente en la displasia ectodérmica hidróticaanhidrótica), prótesis parciales dentomucosoportada de resina, o removibles de cromocobalto, prótesis fija provisional, con la problemática que presentan, a veces, debido a la forma de los dientes y el poco desarrollo de la cresta alveolar edéntula, hasta las más actuales de prótesis cementada sobre implantes, pasando por las coronas cerámicas y reconstrucciones con composites e incluso tratamiento ortodóncico (con aparatología fija multibrackets que permitirá modificar la posición de dientes, crear espacios que nos puedan interesar para confeccionar prótesis implantosoportadas o mucoimplantosoportadas, cerrar diastemas, ayudándose en ocasiones de implantes o minitornillos de titanio para realizar algunos movimientos, etc.). ${ }^{13-18}$

Desde nuestro punto de vista, la utilización de prótesis parciales de acrílico es una alternativa interesante y práctica que permite de una manera relativamente fácil, aceptable, rápida, económica,... dar una solución a la estética, funcionalidad y rehabilitación oral de estos pacientes con marcado edentulismo, que va a permitir una mejora de la vida de relación, una óptima integración social y, así mismo, nos permite una estimulación de los procesos alveolares para un pos- terior tratamiento con una prótesis soportadas sobre implantes como solución más estética y estable para estos pacientes con múltiples agenesias dentales.

\section{BIBLIOGRAFÍA}

1. Armijo M, Ortega RM. Atrofias. En Armijo M, Camacho F. Tratado de Dermatología vol I. Madrid: Aula Médica; 1998. p. 322-5.

2. Pinheiro M, Freire-Maia N. Ectodermal dysplasias: a clinical classification and a causal review. Am J Med Genet 1994; 53: 153-62.

3. Lamartine J. Towards a new classification of ectodermal dysplasias. Clin Exp Dermatol 2003; 28 : 351-5.

4. Faiez N, Angmar-Mansson and cols. Oligodontia of the permanent dentition in two sisters with polycystic ovarian syndrome. Oral Surg Oral Med Oral Pathol Oral Radio End 1997; 87: 368-71.

5. Tsai PF, Chiou HR, Tseng ChCh. Oligodontia-A case report. Quint Int 1998; 29: 191-3.

6. Avilés JA, Hernanz JM, Huerta M. Displasia ectodérmica. Acta Pediatr Esp 2003; 61: 474-5.

7. Peñarrocha M, Sanchís JM, Frutos JR; Estrela F; Pi J. Rehabilitación oral con implantes dentales en un niño con displasia ectodérmica anhidrótica. Medicina Oral 2000; 5: 283-6.

8. Alvarez E, Ruiz JA, Espinal GE, Tibacan DC, Linares EK, Sáez MR. Displasia ectodérmica hipohidrótica: caractrísticas dentales en 11 casos. Arch Odontoestomatol 2004;20:455-62.

9. Pipa A, Gonzalez M, Lopez-Arranz E, Fernández J. Incontinencia pigmentaria. Consideraciones odontoestomatológicas: Profilaxis y terapéutica. Av Odontoestomatol (en prensa).

10. Schnur RE. Genodermatoses 2003-2004. Curr Opin Pediatr 2004; 16: 678-88.

11. Lamartine J, Essenfelder GM, Kibar Z, et al. Mutations in GJB6 cause hidrotic ectodermal dysplasia.Nat Genet 2000; 26:142-4.

12. Pavarina AC, Machado AL, Vergani CE, Gianpaolo ET. Overlay removabe partial dentures for a patient with ectodermal dysplasia: a clinical report. J Prosthet Dent 2001;86:574-7.

13. Suri S, Carmichael R, Tompson B. Simultaneous functional and fixed appliance therapy for growth modification and dental alignment prior to prosthetic habilitation in hypohidrotic ectodermal 
dysplasia: a clinical report. J Prosthet Dent 2004; 92:428-33.

14. Della D, Alonso AB, Cople L, Farinhas A. Alternative rehabilitation treatment for a patient with ectodermal dysplasia. J Clin Pediatr Dent 2004; 28: 103-6.

15. Peñarrocha M, Uribe R, Rambla J, Guarinos J. Fixed rehabilitation of a patient with hypohidrotic ectodermal dysplasia using zygomatic implants. Oral Surg Oral Med Oral Pathol Oral Radiol Endod 2004; 98: 161-5.

16. Hutch KC, Sagner T, Hickel R, Rudzki-Jason I. Interdisciplinary rehabilitation and prevention in a case with early and extensive loss of primary teeth. J Clin Pediatr Dent 2002; 26: 125-9.

17. Guckes AD, Scurria MS, King TS, McCarthy GR, Brahim JS. Prospective clinical trial of dental implants in persons with ectodermal dysplasia. J Prosthet Dent 2002; 88: 21-5.

18. Bergendal B. The role of prosthodontists in habilitation and rehabilitation in rare disorders: the ectodermal dysplasia experience. Int $\mathrm{J}$ Prosthodont 2001; 14: 466-70.

\section{CORRESPONDENCIA}

Adolfo Pipa Vallejo

Escuela de Odontología

Catedrático José Serrano, s/n

33006 Oviedo

Tfno.: 985103620

Fax: 984192261

Correo electrónico: pipaadolfo@uniovi.es 